\title{
ANÁLISIS ECONÓMICO DE LA PRODUCCIÓN DE ORÉGANO (Origanum vulgare L.) EN LOS ASENTAMIENTOS DE LA YARADA DEL DISTRITO DE TACNA
}

\author{
ECONOMIC ANALYSIS OF THE PRODUCTION OF OREGANO (Origanum vulgare \\ L.) IN THE ESTABLISHMENTS OF LA YARADA IN THE DISTRICT OF TACNA
}

\author{
${ }^{1}$ Francisco Condori Tintaya; ${ }^{2}$ Hernan Hurtado Hurtado
}

\begin{abstract}
RESUMEN
El orégano es uno de los cultivos agroexportables de gran importancia en la Región Tacna, producido en altitudes de 2000 a 3500 m.s.n.m.; Sin embargo, a partir del año 2011 hacia adelante, este cultivo se adaptó en valles de costa como La Yarada y Los Palos, con una superficie mayor a 300 has. La variables analizadas fueron la rentabilidad (Y) versus los factores productivos $(X)$; para tal efecto, se utilizó indicadores de evaluación económica $(V A N, T I R, B / C, P E)$ para medir su nivel de rentabilidad, utilizando estados financieros como Estado de pérdidas y ganancias y flujo de caja. Se aplicó un cuestionario a 109 productores de las zonas de La Yarada y Los Palos. De los análisis practicados, se determinó que la rentabilidad media en una hectárea de orégano/año son los siguientes: $V A N=S / .101$ 103,08; TIR $=168 \% ; B / C=2,01$. Estos indicadores señalan que la producción de orégano es sumamente rentable, bajo el precio actual S/. 7,00 soles el kg de orégano seco; sin embargo, se ha determinado que la rentabilidad puede variar debido a la volatilidad del precio.
\end{abstract}

Palabras Claves: Costos, beneficios, rentabilidad, sensibilidad.

\begin{abstract}
Oregano is one of the agroexportables crops of great importance in Tacna Region, produced at altitudes from 2000 to 3500 meters above sea level; however, from 2011 forward, this crop was adapted in valleys on the coast as la Yarada and Los Palos, with one area greater than 300 hectares. The variables analyzed were profitability (Y) versus the productive factors (Xi); for this purpose, economic evaluation indicators $(N P V, I I R, B / C, P E)$ were used to measure their level of profitability, using financial statements profit, loss statement and cash flow. A questionnaire was administered to 109 producers in areas of La Yarada and Los Palos. From the analysis, it was determined that the profitability of media in a hectare of oregano/year are as follows: $g o=N P V=S / .101103 .08$; $I I R=168 \% ; B / C=2.01$. These indicators show that the production of oregano is highly profitable, under the current price $S / .7,00$ soles per kg of dried oregano; however, it has been determined that profitability can vary due to the volatility of the price.
\end{abstract}

Key Words: Costs, benefits, cost, sensitivity

\section{INTRODUCCIÓN}

En los últimos años, el cultivo del orégano viene ampliando su frontera agrícola en regiones como Arequipa, Moquegua, Ancash y Huaraz. A pesar de esto la región Tacna produce un volumen promedio equivalente al $62 \%$ de la oferta nacional (MINAGRI, 2012). El cultivo de esta especie en Tacna tiene una tradición de más de 60 años; sin embargo, en los últimos 20 años tuvo un crecimiento sostenible llegando a ser cultivado por más de 1250 pequeños productores, localizados fundamentalmente en los valles interandinos de la región en una extensión aproximada de 1500 has.

El orégano cobra importancia alimenticia por ser un condimento natural muy apreciado. A nivel industrial se usa en perfumería y productos de tocador. En medicina, en la elaboración de anestesias, sedantes, antiespasmódicos, antirreumáticos y expectorantes. En la industria de la carne es utilizado como esencia y condimento natural (Silva, 2012)

La creciente demanda de este producto sobre todo en su estado de flor ha permitido experimentar su producción en zonas de costa como la Yarada y los Palos con muy buenos resultados, tal es así que, en la actualidad se produce una superficie mayor a 300 has.

El conocimiento de los costos de producción bajo los distintos sistemas de conducción y su rentabilidad, podría permitir que agricultores de otras zonas como el valle viejo del sector Uchusuma puedan diversificar su cédula de cultivos. Por esta razón, la presente investigación planteó como objetivo general analizar los costos de producción y rentabilidad del orégano producido en las zonas de la Yarada y los Palos. 
Condori F, y Hurtado H., Análisis Económico de la Producción de Orẻgano (Origanum vilgare L.) en los asentamientos de la Yarada del distrito de Tacna

\section{MATERIALES Y MÉTODOS}

\section{1. Ámbito de estudio}

El estudio se desarrolló en las zonas agrícolas de la Yarada y los Palos, que son sectores productivos de la cuenca del río Caplina, localizado al extremo sur del Perú, entre las coordenadas $15^{\circ} 17^{\prime}$ y $18^{\circ} 18^{\prime}$ de la latitud Sur y $69^{\circ} 28^{\prime}$ y $71^{\circ} 23^{\prime}$ de la latitud Oeste, en la Región Costa.

\subsection{Variables de la investigación}

Variable dependiente $\left(\mathrm{Y}_{j}\right)$ :

Rentabilidad, expresada en términos de VAN, TIR y B/C.

Variables independientes $(\mathrm{X})$ :

$\mathrm{X}_{1}=$ Inversión inicial (Nuevos soles/ha)

$\mathrm{X}_{2}=$ Costo de producción (Nuevos soles $/ \mathrm{ha}$ )

$\mathrm{X}_{3}=$ Precio del producto (nuevos soles $/ \mathrm{kg}$ )

$\mathrm{Y}_{\mathrm{i}}=\int\left(\mathrm{X}_{1}+\mathrm{X}_{2}+\mathrm{X}_{3}\right)$

\subsection{Población}

La población (N), está compuesto por los usuarios de riego de las localidades de la Yarada y los Palos, que en total son 1137 regantes.

\subsection{Muestra}

El método de muestreo utilizado fue el de aleatorio simple, habiendo tomado como elementos de muestra agricultores que tenían plantaciones de orégano en producción y en crecimiento, por tanto el tamaño fue $\mathrm{n}=$ 109 muestras.

\section{RESULTADOS Y DISCUSIÓN}

\subsection{Descripción de la producción de orégano en el ámbito de estudio.}

Las mayores plantaciones de orégano están localizadas en los Palos en los sectores comprendidos: "Juan Velazco Alvarado" y "Los Palos", y en La Yarada, en los sectores de "La Esperanza" " "Los Olivos".

De acuerdo a la información recabada de los trabajos de campo, el 54,1\% de los predios manifestaron tener una extensión de orégano entre menor o igual a 02 has, el $36,7 \%$ de los predios tiene entre mayor de 02 a 04 has de tierras con plantaciones de orégano, el restante manifiesta tener más de 04 has de tierras con plantaciones de orégano hasta un máximo de 10 Has. Es importante destacar que, aproximadamente el $50 \%$ de los agricultores encuestados no sólo tienen plantaciones de orégano en producción, sino que también tienen plantaciones de orégano en reciente crecimiento y algunos cuentan con terrenos preparados para nuevas plantaciones de orégano.

Los análisis estadísticos practicados indican que, en términos generales, en el ámbito de estudio, cada usuario tiene en promedio 2,65 has de tierras con plantaciones de orégano, con una desviación típica de 1,71 has y una varianza de 2,94 has. Este último estadístico indica que existe una varianza significativa es decir que el rango de la desviación está comprendida en una diferencia de 9,5 has, es decir entre los que tienen menos y los que tienen más

Entre los predios que tienen plantaciones de orégano, el $80,7 \%$ tienen al menos 02 has de orégano en producción, el 16,5\% tienen entre 02 a 04 has de orégano en producción, y aproximadamente $3 \%$ de los predios analizados tienen entre 04 a 06 has de orégano en plena producción. Ver Tabla $\mathrm{N}^{\circ} 01$

Generalmente los que tienen mayor superficie con plantaciones de orégano en producción son aquellos que tempranamente iniciaron con este cultivo, por lo tanto, son productores más especializados y con mucha solvencia económica para ampliar los nuevos campos de orégano.

Tabla $\mathrm{N}^{\circ} 01$. Total has orégano en producción por parcela

\begin{tabular}{cccc}
\hline & & Frecuencia & Porcentaje \\
\hline Has & $<=$ a 2 & 88 & 80,7 \\
& 2,1 a 4 & 18 & 16,5 \\
4,1 a 6 & 3 & 2,8 \\
& Total & 109 & 100,0 \\
\hline
\end{tabular}

Fuente: Elaboración propia

\subsection{Algunas prácticas agronómicas para la producción del orégano en el ámbito de estudio.}

La preparación del terreno es una labor fundamental, según Álvarez (2005), los productores asignan terrenos trabajados con bajo contenido de sales, de textura franco arenoso, y por el tipo de suelo agregan gran cantidad de materia orgánica, incluso trasladan este insumo (M.O.) desde las zonas alto andinas de Tacna y Puno (estiércol del ganado camélidos) para evitar la proliferación de malezas.

Los surcos se realizan con un distanciamiento entre 0,60 y $0,80 \mathrm{~m}$, y en el lomo de estos surcos se realiza las plantaciones. Los esquejes de orégano previamente son tratados y se utiliza un promedio de 65000 esquejes por ha. Los riegos son frecuentes en algunos casos diarios, los fertilización lo realizan mayormente por el sistema de riego utilizando abonos solubles.

Un grupo poco significativo de productores aplica solamente fertilizantes, en la mayoría de los casos manifiestan que utilizan ambos tipos de insumos. Ver Tabla $\mathrm{N}^{\circ} 02$.

Tabla $\mathrm{N}^{\circ} 02$. Tipo de abonamientos aplicados en la producción de orégano.

\begin{tabular}{llcc}
\hline & & Frecuencia & Porcentaje \\
\hline Válidos & Fertilizantes & 21 & 19,3 \\
& Ambos tipos & 88 & 80,7 \\
& Total & 109 & 100,0 \\
\hline
\end{tabular}

Fuente: Elaboración propia

Para el control de las plagas y enfermedades aplicaron productos químicos en el $98,2 \%$ de los casos, el $1,8 \%$ de productores respondieron que no aplicaron productos químicos, esto posiblemente debido a que las plantaciones aún se encontraban en los primeros estadios de crecimiento. Ver Tabla $\mathrm{N}^{\circ} 03$.

De acuerdo a la información de campo, las aplicaciones se realizaron en el $52 \%$ de los casos cada dos 
Conderi F, y Hurtado H., Análisis Económico de la Producción de Orégano (Origanum vulgare L.) en los asentamientos de la Yarada del distrito de Tacna

semanas, en el $20 \%$ de los casos cada semana y en el $18 \%$ de los casos cada mes.

Tabla $\mathrm{N}^{\circ} 03$. Aplica pesticidas para el control de plagas y enfermedades

\begin{tabular}{llcc}
\hline & & Frecuencia & Porcentaje \\
\hline Válidos & si & 107 & 98,2 \\
& no & 2 & 1,8 \\
& Total & 109 & 100,0 \\
\hline
\end{tabular}

Fuente: Elaboración propia

\subsection{Cosecha}

Las cosechas o cortes del orégano, varían según la época que fueron plantados, generalmente ocurre cada 4 o 5 meses; sin embargo el primer corte toma un mayor tiempo y ocurre a los 7 meses después de ser plantados en campo; es decir las cosechas están distribuidas durante todo el año, no existe una sola época.

De acuerdo a la información de campo, el primer corte lo realizan generalmente en el $52 \%$ entre los meses de julio a setiembre, el segundo corte mayormente entre octubre a diciembre en el $47 \%$ de los casos y el tercer corte lo realizan mayormente entre los meses de abril a junio en el $52 \%$ de los casos.

El desarrollo fenológico de la producción del orégano fue tomado en base a una parcela ubicado en la zona de los Palos, que muestra la siguiente cronología:

\begin{tabular}{|c|c|c|c|c|}
\hline \multicolumn{2}{|c|}{$1 \mathrm{er}$ año 2 cortes } & \multicolumn{3}{|c|}{2 do año 3 cortes } \\
\hline 7 & 4 & 5 & 4 & 4 \\
\hline 2013 & 2013 & 2014 & 2014 & 2015 \\
\hline MAR-SET & OCT-ENE & FEB-JUN & JUL-OCT & NOV-FEB \\
\hline
\end{tabular}

Figura $\mathrm{N}^{\circ} 01$ Cronología cosechas de orégano en zona los Palos.

Fuente: Elaboración propia

De la Figura $\mathrm{N}^{\circ} 01$ se puede deducir que, en el primer año ocurren mayormente solo dos cortes y en el segundo año se pueden obtener hasta tres cortes; para los años siguientes este comportamiento se repite.

El rendimiento que se obtiene del corte por hectárea es muy variable, el mismo que está en función al nivel tecnológico aplicado en su manejo; sin embargo, la información levantada en campo señala que el 33\% de los productores obtienen rendimientos entre $6000 \mathrm{a} 9000 \mathrm{~kg}$ de hoja seca por ha/año, un $31 \%$ obtienen rendimientos entre $3000 \mathrm{a} 6000 \mathrm{~kg}$ de hoja seca/ha/año y un porcentaje del $13 \%$ obtienen entre $9000 \mathrm{a} 12000 \mathrm{~kg} / \mathrm{ha} / \mathrm{año}$.

\subsection{Análisis de costos}

\section{a) Inversiones fijas}

Para medir la rentabilidad se han definido primeramente las inversiones fijas tangibles e intangibles y el capital de trabajo. Para efectos del presente análisis, se ha considerado como inversiones fijas todos los costos que demandaron la instalación del cultivo en campo a partir del cual, todos los siguientes costos se consideraron como capital de trabajo.
Tabla $\mathrm{N}^{\circ} 04$. Costos de inversión para instalar 01 ha de orégano.

\begin{tabular}{|c|c|c|c|c|}
\hline Descripción & Unidad & Cant. & P.Unit & Total \\
\hline \multicolumn{5}{|l|}{ MANO DE OBRA } \\
\hline $\begin{array}{l}\text { Preparación del Terreno } \\
\text { Limpicza, despaje, acantone y } \\
\text { quema }\end{array}$ & jh & 2 & 40 & $\begin{array}{r}1200 \\
80\end{array}$ \\
\hline Incorporación de Abono Orgánico & ih & 10 & 40 & 400 \\
\hline Tapado y Nivelación & jh & 10 & 40 & 400 \\
\hline Tendido de Cintas de Riego & ih & 6 & 40 & 240 \\
\hline Riego, remojo & ih & 2 & 40 & 80 \\
\hline \multicolumn{5}{|l|}{ Siembra o Trasplante } \\
\hline $\begin{array}{l}\text { Desinfección o tratamiento } \\
\text { fitosanitario }\end{array}$ & jh & 1 & 40 & 40 \\
\hline Trasplante & ih & 40 & 40 & 1600 \\
\hline Retrasplante & ith & 15 & 40 & 600 \\
\hline \multicolumn{5}{|l|}{ MAQUINARIA } \\
\hline Arado & $\mathrm{H}-\mathrm{M}$ & 3 & 70 & 210 \\
\hline Nivelado & $\mathrm{H}-\mathrm{M}$ & 2 & 70 & 140 \\
\hline Surqueo & $\mathrm{H}-\mathrm{M}$ & 2 & 70 & 140 \\
\hline \multicolumn{5}{|l|}{ INSUMOS } \\
\hline Esquejes & Esq & 65000 & 0,038 & 2470 \\
\hline \multicolumn{5}{|l|}{ TRANSPORTE } \\
\hline $\begin{array}{l}\text { Transporte de orégano de } \\
\text { propagación }\end{array}$ & Global & 1 & 150 & 150 \\
\hline Transporte de Abono Orgánico & Global & 1 & 50 & 50 \\
\hline \multicolumn{4}{|l|}{ EQUIPO DE RIEGO } & 4000 \\
\hline Elementos de equipo de riego & Global & 1 & 4000 & 4000 \\
\hline \multicolumn{5}{|l|}{ OTROS } \\
\hline $\begin{array}{l}\text { Festejos instalaciòn de } \\
\text { órgano(tradición) }\end{array}$ & Global & 1 & 200 & 200 \\
\hline Asistencia técnica & Global & 1 & 600 & 600 \\
\hline \multicolumn{5}{|l|}{$\begin{array}{l}\text { Valor alquiler terreno/vida útil del } \\
\text { orégano }\end{array}$} \\
\hline TOTAI.S/. & & & & 17040 \\
\hline
\end{tabular}

Fuente: Elaboración propia

Las inversiones fijas tangibles están representadas por el costo del equipo de riego y las plántulas de orégano o esquejes (ver Tabla $\mathrm{N}^{\circ} 04$ ), representando un total de $\mathrm{S} / .6470$ nuevos soles; en ambos casos se ha estimado que su vida útil es solo de 03 años, por lo tanto no tiene valor de desecho o salvamento. $\mathrm{La}$ depreciación de ambos activos se ha calculado por el método lineal.

En cuanto a los activos fijos intangibles, estos representan un valor de S/. 10570 nuevos soles (ver Tabla $\mathrm{N}^{\circ} 04$ ), y está representado por todos los costos que son fungibles en la etapa de instalación del orégano como: mano de obra, costo servicio de maquinaria, servicio de transportes y rubros considerados como otros; y su recuperación se realizó por el método de las amortizaciones en un horizonte de 03 años.

\section{b) Capital de trabajo}

Se ha considerado como capital de trabajo todos los costos y gastos necesarios para garantizar el ciclo productivo del orégano, es decir desde que el campo está instalado hasta las acciones que demandan la fase de la comercialización; por lo tanto estos costos y gastos están 
Conderi F. y Hurtado H., Análisis Económico de la Producción de Orégano (Origanum vulgare L.) en los asentamientos de la Yarada del distrito de Tacna

agrupados en costos directos e indirectos que hacen el costo de producción y los gastos operativos que están representados en gastos de administración y ventas.

Por otra parte, el total de los costos fueron analizados si tienen comportamiento fijo o variable respecto al volumen de producción, datos que más adelante permitió calcular el punto de equilibrio.

La cantidad de recursos económicos en efectivo que se requirió como capital de trabajo para los años 01 y 02 , se han calculado utilizando el método del periodo de desfase o ciclo productivo. (ver Tabla $\mathrm{N}^{\circ} 05$ )

Tabla $\mathrm{N}^{\circ} 05$. Costos de producción / ha/corte.

\begin{tabular}{|c|c|c|c|c|}
\hline Descripción & 1er corte & 2 do corte & 3er corte & TOTAL/año \\
\hline Año 01 & 12607,40 & 10982,00 & 0,00 & 23589,40 \\
\hline $\begin{array}{c}\text { uente: Elabor } \\
\text { Año } 02\end{array}$ & $\begin{array}{c}\text { ón propia } \\
\text { if } 982,00\end{array}$ & 10982,00 & 10982,00 & 32946,00 \\
\hline
\end{tabular}

\section{Cálculo de capital de trabajo:}

$\mathrm{KW}=\mathrm{CP} * \mathrm{COPD}$

Dónde:

$\begin{array}{ll}\mathrm{KW} & =\text { Capital de trabajo } \\ \mathrm{CP} & =\text { Ciclo productivo }\left(\mathrm{N}^{\circ} \text { de días) }\right. \\ \mathrm{COPD} & =\text { Costo operación promedio día }(\mathrm{S} / .)\end{array}$

Capital de trabajo año 01:

$$
\begin{array}{ll}
\mathrm{COPD} & =23589,40 / 365 \text { dias }=64,6284 / \text { día } \\
\mathrm{CP} & =\text { Promedio } 165 \text { dias } \\
\mathrm{KW} & =165 \text { dias } * 64,6284 / \text { día } \\
\mathrm{KW}_{1} & =10663,68 \text { nuevos soles }
\end{array}
$$

\section{Capital de trabajo año 02:}

$$
\begin{array}{ll}
\mathrm{COPD} & =32940 / 365 \text { días }=90,2630 / \mathrm{dia} \\
\mathrm{CP} & =\text { Promedio } 120 \text { días }
\end{array}
$$

$\mathrm{KW}=120$ días $* 90,2630 /$ día

$\mathrm{KW}_{2} \quad=10831,56$ nuevos soles

\section{Incremento entre año 01 y año 02:}

$$
\mathrm{KW}_{2} \mathrm{KW}_{1}=167,88 \text { nuevos soles }
$$

\subsection{Análisis económico}

\section{a) Estado de pérdidas y ganancias}

El análisis económico para la presente investigación, está expresado en el estado de pérdidas y ganancias, análisis que se procesó para una campaña (corte) y también para un periodo anual. (ver tablas $\mathrm{N}^{\circ} 08$ y $\mathrm{N}^{\circ} 09$ )

La información base utilizada para los análisis económicos es el que está contenido en las tablas $\mathrm{N}^{\circ} 06 \mathrm{y}$ $\mathrm{N}^{\circ} 07$.

El análisis de pérdidas y ganancias fue también calculado por cada corte o campaña, así como en forma anualizada. En la Tabla $\mathrm{N}^{\circ} 08$, se aprecia que, en el primer año de operación, solo se obtuvo dos cortes de orégano; sin
Tabla $\mathrm{N}^{\circ} 06$. Resultados primer año de producción

\begin{tabular}{lrrrr}
\hline Descripción & 1er corte & 2do corte & 3er corte & TOTAL/an̂o \\
\hline Rdto bruto $(\mathrm{kg} / \mathrm{ha})$ & 4000,00 & 5000,00 & 0,00 & 9000,00 \\
Mermas $(2 \%)$ & 80,00 & 100,00 & 0,00 & 180,00 \\
Rdto neto $(\mathrm{kg} / \mathrm{ha})$ & 3920,00 & 4900,00 & 0,00 & 8820,00 \\
P.U. venta $(\mathrm{kg})$ & 7,00 & 7,00 & 0,00 & \\
Ingreso total & 27440,0 & 34300,0 & 0,00 & 61740,00 \\
Costo Total & 12607,4 & 10982,0 & 0,00 & 23589,40
\end{tabular}

Fuente: Elaboración propia

Tabla N07. Resultados segundo año de producción

\begin{tabular}{lrrrr}
\hline \multicolumn{1}{c}{ Descripción } & 1er corte & 2do corte & 3er corte & TOTAL/año \\
\hline Rdto bruto $(\mathrm{kg} / \mathrm{ha})$ & 5000,00 & 5000,00 & 5000,00 & 15000,00 \\
Mermas $(2 \%)$ & 100,00 & 100,00 & 100,00 & 300,00 \\
Rdto neto $(\mathrm{kg} / \mathrm{ha})$ & 4900,00 & 4900,00 & 4900,00 & 14700,00 \\
Precio u. venta $(\mathrm{kg})$ & 7,00 & 7,00 & 7,00 & \\
Ingreso total & 34300,00 & 34300,00 & 34300,00 & 102900,00 \\
Costo Total & 10982,00 & 10982,00 & 10982,00 & 32946,00 \\
\hline
\end{tabular}

Fuente: Elaboración propia

Tabla $N^{\circ} 08$. Estado de pérdidas y ganancias 1er año

\begin{tabular}{lrrr}
\hline \multicolumn{1}{c}{ Concepto } & 1er corte & 2do corte & TOTAL S/. \\
\hline Ingreso por Ventas & 27440,00 & 34300,00 & 61740,00 \\
Costos de Producción & 12007,40 & 10382,00 & 22389,40 \\
UTHIDAD BRUTA & 15432,60 & 23918,00 & 39350,60 \\
Gastos de Adm. & 500,00 & 500,00 & 1000,00 \\
Gastos de ventas & 100,00 & 100,00 & 200,00 \\
UT7I.JDAD OPER ATIVA & 14832,60 & 23318,00 & 38150,60 \\
Ingresos Financicros & 0,00 & 0,00 & 0,00 \\
Otros Ingresos & 0,00 & 0,00 & 0,00 \\
U. A. DE INTER. E IMPTOS & 14832,60 & 23318,00 & 38150,60 \\
Intereses del préstamo & 0,00 & 0,00 & 0,00 \\
Impuestos (IR=15\%) & 0,00 & 0,00 & 0,00 \\
UTIIIIDAD NETA & 14832,60 & 23318,00 & 38150,60 \\
\hline Fuente: Elaboración propia & & &
\end{tabular}

Tabla $\mathrm{N}^{\circ} 09$. Estado de pérdidas y ganancias 2 do y $3 \mathrm{er}$ año.

\begin{tabular}{lrrrr}
\hline \multicolumn{1}{c}{ Concepto } & 1er corte & 2do corte & 3er corte & TOTAL S/. \\
\hline Ingreso por Ventas & 34300,00 & 34300,00 & 34300,00 & 102900,00 \\
Costos de Producción & 10982,00 & 10982.00 & 10982,00 & 32946,00 \\
UTHLIDAD BRUTA & 23318,00 & 23318,00 & 23318,00 & 69954,00 \\
Gastos de Adm. & 500,00 & 500,00 & 500,00 & 1500,00 \\
Gastos de ventas & 100,00 & 100,00 & 100,00 & 300,00 \\
UTHLIDAD OPER ATTVA & 22718,00 & 22718,00 & 22718,00 & 68154,00 \\
Ingresos Financieros & 0,00 & 0,00 & 0,00 & 0,00 \\
Otros Ingresos & 0,00 & 0,00 & 0,00 & 0,00 \\
& & & & \\
U. A. DE INTE. E IMPTOS, & 22718,00 & 22718,00 & 22718,00 & 68154,00 \\
Intcreses del préstamo & 0,00 & 0,00 & 0,00 & 0,00 \\
Impuestos & 0,00 & 0,00 & 0,00 & 0,00 \\
UTHLID AD NETA & 22718,00 & 22718,00 & 22718,00 & 68154,00 \\
\hline Fuente: Elaboración propia & & & &
\end{tabular}

embargo, los siguientes años el número de cortes se incrementa a tres por año.

En la Figura $\mathrm{N}^{\circ} 01$, se muestra que por ser el primer corte el ciclo de producción tarda aproximadamente siete meses y el siguiente aproximadamente cinco meses; razón por el cual en el primer año se obtiene solo dos cortes al año.

De acuerdo a la información de campo en el segundo año de operación la producción logra estabilizarse, es decir, se obtiene tres cortes, pudiendo los rendimientos ser mayores. 


\section{b) Punto de equilibrio}

El punto de equilibrio depende de la cuantía de la variable ingreso total $(\mathrm{YT})$, costos fijos $(\mathrm{CF})$ y variables (CV) en la estructura de costos, y este indicador se ha calculado para cada corte o cosecha y también para cada periodo anual.

En las tablas $\mathrm{N}^{\circ} 10$ y 11 , se muestran los resultados del análisis, donde el punto de equilibrio para el primer corte es mayor a los obtenidos en los siguientes cortes, puesto que en éste periodo ocurren bajos ingresos y mayores costos; el punto de equilibrio se reduce en los siguientes cortes debido a que la producción se incrementa.

Analizando la evolución de la producción de orégano a partir del segundo año, se observa que alcanza el umbral de la producción, por tanto la cantidad de orégano seco que se tiene que producir por corte/ha para cubrir los costos fijos y variables es de $500,38 \mathrm{~kg} /$ ha y una producción anual de $1501,13 \mathrm{~kg} / \mathrm{ha}$.

Tabla $\mathrm{N}^{\circ} 10$. Punto de equilibrio 1er año de producción

\begin{tabular}{lrrr}
\hline \multicolumn{1}{c}{ Concepto } & 1er corte & 2do corte & TOTAL S/. \\
\hline Ingreso Total (YT) & 27440,00 & 34300,00 & 61740,00 \\
Costo fijo (CF) & 3126,40 & 2652,00 & 5778,40 \\
Costo Variable (CV) & 9481,00 & 8330,00 & 17811,00 \\
Costo total (CT) & 12607,40 & 10982,00 & 23589,40 \\
Cantidad producida (Q) & 3920,00 & 4900,00 & 8820,00 \\
Precio de venta (P) & 7,00 & 7,00 & 7,00 \\
Costo variable unitario (Cvu) & 2,42 & 1,70 & 2,02 \\
Punto de equilibrio (PE) & 682,41 & 500,38 & 1160,18 \\
\hline
\end{tabular}

Fuente: Elaboración propia

Tabla $\mathrm{N}^{\circ}$ 11. Punto de equilibrio 2 do año de producción

\begin{tabular}{lrrrr}
\hline \multicolumn{1}{c}{ Concepto } & 1er corte & 2do corte & 3er corte & TOTAL S/. \\
\hline Ingreso Total (YT) & 34300,00 & 34300,00 & 34300,00 & 102900,00 \\
Costo fijo (CF) & 2652,00 & 2652,00 & 2652,00 & 7956,00 \\
Costo Variable (CV) & 8330,00 & 8330,00 & 8330,00 & 24990,00 \\
Costo total (CT) & 10982,00 & 10982,00 & 10982,00 & 32946,00 \\
Cantidad (Q) & 4900,00 & 4900,00 & 4900,00 & 14700,00 \\
Precio de venta (P) & 7,00 & 7,00 & 7,00 & 7,00 \\
Costo V. unitario (Cvu) & 1,70 & 1,70 & 1,70 & 1,70 \\
Punto de equilibrio (PE) & 500,38 & 500,38 & 500,38 & $\mathbf{1 5 0 1 , 1 3}$ \\
\hline
\end{tabular}

Fuente: Elaboración propia

En la Figura $\mathrm{N}^{\circ} 02$ se muestra el punto de equilibrio para el segundo año, considerando que en este año el orégano logra una estabilización de su producción.

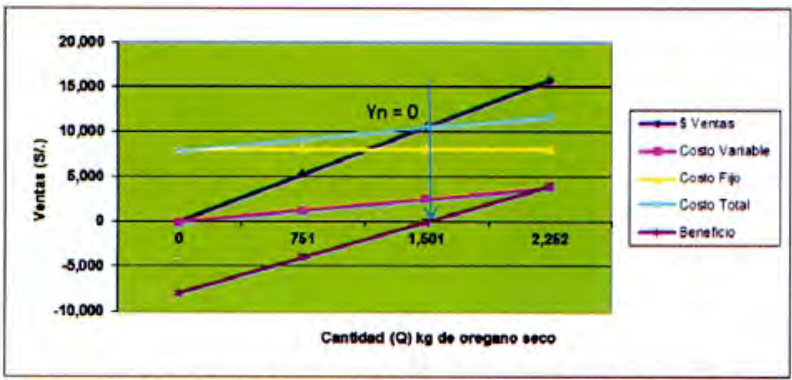

Figura $\mathrm{N}^{\circ}$ 02. Punto de equilibrio 2do año de producción

\subsection{Análisis de rentabilidad}

La rentabilidad de la producción del orégano en la Yarada y los Palos, se ha medido en términos de Valor Actual Neto (VAN), Tasa interna de retorno (TIR), relación Beneficio - Costo $(B / C)$, en consecuencia el análisis se partió de los siguientes supuestos:

Flujo de caja: Para la elaboración del flujo de caja, se ha considerado un momento cero, donde se han registrado todas las inversiones, y los momentos 1,2 , y 3 se han registrado los movimientos de entradas y salidas operativas anualizadas.

Horizonte de evaluación: Para definir el horizonte de la evaluación, se ha tomado como criterio la vida útil de los activos más importantes de la actividad. En este caso, el sistema de riego (cintas de riego) y vida útil de las plantaciones de orégano; en ambos casos, de acuerdo a la experiencia de los productores indican que la vida útil de ambos activos es de tres años; por lo tanto el horizonte de evaluación se definió para ese periodo.

Tasa de Descuento (TD): La tasa de descuento es el costo de oportunidad del inversionista o la tasa de rentabilidad mínima exigida a inversión, y esto se ha calculado en el $18 \%$.

En la tabla $\mathrm{N}^{\circ} 12$ se presenta el flujo de caja económico sobre el cual se calcularon los indicadores de evaluación como el VAN, TIR, y B/C.

Tabla N¹2. Flujo de caja económico

\begin{tabular}{|c|c|c|c|c|}
\hline \multirow{2}{*}{ DESCRIPCIÓN } & \multicolumn{4}{|c|}{ Años } \\
\hline & 0 & 1 & 2 & 3 \\
\hline Ingresos afectos a impuestos & 0,00 & 61740,00 & 102900,00 & 102900,00 \\
\hline Egresos afectos a impuestos & 0,00 & 23589,40 & 34746,00 & 34746,00 \\
\hline Gastos no desembolsables & 0,00 & 5680,00 & 5680,00 & 5680,00 \\
\hline Utilidad antes de imprestos & 0,00 & 32470,60 & 62474,00 & 62474,00 \\
\hline Impuestos (IR 30\%) & 0,00 & 0,00 & 0,00 & 0,00 \\
\hline Utilidad desp de impuestos & 0,00 & 32470,60 & 62474,00 & 62474,00 \\
\hline $\begin{array}{l}\text { Ajuste por gastos no } \\
\text { desembolsables }\end{array}$ & 0,00 & 5680,00 & 5680,00 & 5680,00 \\
\hline $\begin{array}{l}\text { Egresos no afectos a } \\
\text { impuestos }\end{array}$ & 27703,68 & 167,88 & 0,00 & 0,00 \\
\hline Be no afectos a impuestos & 0,00 & 0,00 & 0,00 & 10663,68 \\
\hline $\begin{array}{l}\text { FLUJO DE CAJA } \\
\text { ECONOMICO }\end{array}$ & $-27703,68$ & 37982,72 & 68154,00 & 78817,68 \\
\hline
\end{tabular}

La evaluación aplicada sobre el flujo de caja económico indica que, en términos de valor actual neto $(\mathrm{VAN}=\mathrm{S} / .101403,08)$, la producción de orégano en una hectárea permite primeramente recuperar la inversión anual, en segundo lugar, asegura una rentabilidad igual a su tasa de descuento $18 \%$, y en tercer lugar, permite incrementar el patrimonio del inversionista en S/. 101403,08 nuevos soles al final del horizonte de evaluación. Este resultado VAN $>0$, indica que la actividad económica fue sumamente conveniente.

Por otra parte, en términos de tasa interna de retorno $(T I R=168 \%)$, significa que la producción de orégano en una hectárea, durante el horizonte de evaluación generó una rentabilidad promedio de $168 \%$, valor que al ser TIR > T.D.; la inversión fue sumamente rentable.

La relación Beneficio - Costo $(B / C=2,01)$, señala que por cada unidad monetaria invertida en la producción del orégano, este permitió un retorno de S/. 2,01 nuevos soles. Este resultado $\mathrm{B} / \mathrm{C}>1$, significa que la actividad económica fue sumamente beneficiosa. Este resultado contrastado con los obtenidos en las zonas interandinas de la región Tacna $(B / C=0,8$ a 1,57) la producción de orégano en las zonas de la Yarada y los Palos es más rentable (Paria, 


\subsection{Análisis de sensibilidad}

Para practicar el análisis de sensibilidad, se ha considerado como variables cambiantes la producción/ha/año y el precio de venta por kilogramo de orégano seco (Tabla $\mathrm{N}^{\circ} 13$ ).

El escenario que se analizó en el presente estudio, fue considerando una producción por ha/año de $14700 \mathrm{~kg}$ de orégano seco y su venta a un precio de S/. 7 nuevos soles; bajo estos supuestos, la rentabilidad fue de $168 \%$; sin embargo el análisis demuestra que, en un supuesto que el precio de venta baje, la rentabilidad mínima aceptable se localiza a un precio de $\mathrm{S} / .3$ nuevos soles, pero produciendo $18000 \mathrm{~kg}$, en esta intersección la rentabilidad es de $28,8 \%$, si se produce menos $(14700 \mathrm{~kg})$ al mismo precio, la actividad deja de ser rentable (TIR $=5,9 \%$ ), este valor es menor a su T.D. o costo de oportunidad (18\%) del inversionista, por tanto la producción de orégano dejaría de ser una actividad rentable.

Tabla $N^{\circ} 13$. Resultado de análisis de sensibilidad

\begin{tabular}{|c|c|c|c|c|c|c|}
\hline \multicolumn{7}{|c|}{ Producción de orégano por ha/año } \\
\hline & $168 \%$ & 6000 & 8820 & 12000 & 14700 & 18000 \\
\hline \multirow{9}{*}{ 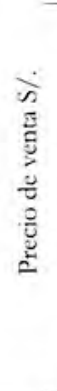 } & 2,00 & & & & $.58,24 \%$ & $-29,39 \%$ \\
\hline & 3,00 & & $-74,65 \%$ & $-19,03 \%$ & $5,97 \%$ & $28,80^{\circ} \%$ \\
\hline & 4,00 & & $-8,27 \%$ & $29,75 \%$ & $52,46 \%$ & $74,96 \%$ \\
\hline & 5,00 & $-16,47 \%$ & $36,76 \%$ & $71,04 \%$ & $93,43 \%$ & $116,40 \%$ \\
\hline & 6,00 & $32,99 \%$ & $76,09 \%$ & $109,12 \%$ & $131,67 \%$ & $155,31 \%$ \\
\hline & 7,00 & $73,64 \%$ & $112,87 \%$ & $145,44 \%$ & $168,31 \%$ & $192,63 \%$ \\
\hline & 8,00 & $111,00 \%$ & $148,24 \%$ & $180,67 \%$ & $203,90 \%$ & $228,87 \%$ \\
\hline & 9,00 & $146,69 \%$ & $182,73 \%$ & $215,17 \%$ & $238,76 \%$ & $264,35 \%$ \\
\hline & 10,00 & $181,38 \%$ & $216,64 \%$ & $249,15 \%$ & $273,09 \%$ & $299,25 \%$ \\
\hline
\end{tabular}

Fuente: Elaboración propia

\section{CONCLUSIONES}

La producción de orégano en las zonas de la Yarada y los Palos, introducido recientemente en la zona a partir del año 2011, por el momento muestra una alta rentabilidad, incluso muy superior a los cultivos agroexportables tradicionales como el olivo, algunos cultivos cucurbitáceos (Sandia, Zapallos, etc.).

Los indicadores de rentabilidad calculados para la producción del orégano fueron: $\mathrm{VAN}=\mathrm{S} / .101403,08$; $\mathrm{TIR}=168 \% ; \mathrm{B} / \mathrm{C}=2,01$. Todos los indicadores calculados $y$ analizados muestran valores muy favorables para los inversionistas; sin embargo, analizando su sensibilidad, el análisis demuestra que la variable crítica es el precio, es decir, mientras se mantenga buen precio $(\mathrm{S} / .7 / \mathrm{kg}$ de orégano seco) la rentabilidad son los que se mostraron; pero si el precio disminuye, lo cual es lo más probable en el tiempo, el cultivo puede mantenerse rentable para la mayoría de productores hasta un precio de $\mathrm{S} / .4$ nuevos soles $/ \mathrm{kg}$, si el precio desciende hasta $\mathrm{S} / .3$ nuevos soles $/ \mathrm{kg}$, entonces la rentabilidad es solo para aquellos productores que tienen la capacidad de producir igual o mayor a $18000 \mathrm{~kg}$ de orégano/ha/año.

\section{RECOMENDACIONES}

Es conveniente continuar con los análisis económicos para el resto de cultivos y de esta manera permitir medir no solo el nivel de rentabilidad, sino fundamentalmente el grado de sensibilidad de la producción frente a posibles variaciones de los precios en el mercado, siendo muy común que se den estas variaciones por efectos de la oferta y demanda.

\section{REFERENCIAS BIBLIOGRÁFICAS}

Alvarez Navarro, Marino, (2005). Orientaciones Generales para la promoción y apoyo a las cadenas Agro productivas en el Perú. Primera Edición DGPA-MINAG, Lima

Minagri (2012), Boletín informativo, Ministerio de Agricultura y Riego-Tacna.

Paria Gallegos, Santos. (2012) Análisis Económico del cultivo de orégano (Origanum vulgare L.) en la Comunidad Campesina de Borogeña, Distrito de Ilabaya, Tesis FCAG/UNJBG.

Silva Vazquez, Ramón (2012) El Orégano (Lippia berlandieri Schauer) una Alternativa Agroindustrial para zonas áridas y semiáridas de México; Centro de Investigación para los Recursos Naturales (CIReNa), México.

\section{Correspondencia:}

Francisco Condori Tintaya: fracontintaya $@$ yahoo.es
Fecha de Recepción: 06/05/2014

Fecha de Aceptación: 18/06/2014 